\title{
Interaction of TATP with Some Group II Metals - A DFT Treatment
}

\section{Lemi Türker}

Department of Chemistry, Middle East Technical University, Üniversiteler, Eskişehir Yolu No: 1, 06800 Çankaya/Ankara, Turkey; e-mail: 1turker@gmail.com; lturker@metu.edu.tr

\begin{abstract}
Triacetonetriperoxide (TATP) is a very sensitive organic peroxide type explosive which attracts the attention of terrorist groups due to its easy synthesis. The present density functional treatment considers the interaction of TATP molecule with certain group II metals at the level of B3LYP/6-311+G(d,p). Composite systems of TATP and Be, 2Be, $\mathrm{Mg}$ and $\mathrm{Ca}$ have been considered. Although, in the case of beryllium composites TATP molecule remains intact, in its $\mathrm{Mg}$ and $\mathrm{Ca}$ composites the rupture of the ring (even in 1:1 composite) occurs. Certain structural, electronic, quantum chemical and some spectral properties of the composites have been obtained and discussed.
\end{abstract}

\section{Introduction}

Organic peroxides are a relatively unexplored class of substances. The most prominent example is acetone peroxide which was synthesized for the first time by the German chemist Richard Wolffenstein in 1895 [1]. His intention was to oxidize Coniin with hydrogen peroxide and used acetone as solvent. The white precipitate formed exhibited to have explosive properties. Detailed investigations concerning the composition of the product were performed later and it was found that the main fraction is a trimeric molecule (triacetone tri peroxide, TATP, 3,3,6,6,9,9-hexamethyl-1,2,4,5,7,8hexoxonane). The trimer is accompanied by a dimer (diacetone di peroxide, DADP) [2]. The ratio of the two possible products are formed depends on the reaction conditions (mostly the concentration of the acid) used during the reaction. Generally concentrated and strong acids favor the formation of DADP whereas lower acid concentrations or acid

Received: September 5, 2021; Accepted: October 3, 2021

Keywords and phrases: TATP; triacetone triperoxide; explosive; beryllium; magnesium; calcium.

Copyright (C) 2022 Lemi Türker. This is an open access article distributed under the Creative Commons Attribution License, which permits unrestricted use, distribution, and reproduction in any medium, provided the original work is properly cited. 
free conditions lead to the formation of TATP. Under the catalysis of tin (IV), a tetrameric form can be isolated [3].

Triacetone triperoxide (TATP), diacetone diperoxide (DADP) and hexamethylene triperoxide diamine (HMTD) (which are sensitive and powerful organic peroxide explosives) have been used by terrorists in the past. They are attractive to terrorists because their syntheses are straightforward, requiring only a few easily obtainable ingredients.

For most peroxides, the general decomposition mechanism is not investigated in detail until now [4]. Only for acetone peroxide deeper studies have been performed. In this case, the main reaction products are carbon dioxide, methane, ethane, ethylene and acetone and no significant amount of heat is released [5]. Under a nitrogen atmosphere, no flame can be observed [5] (entropic detonation).
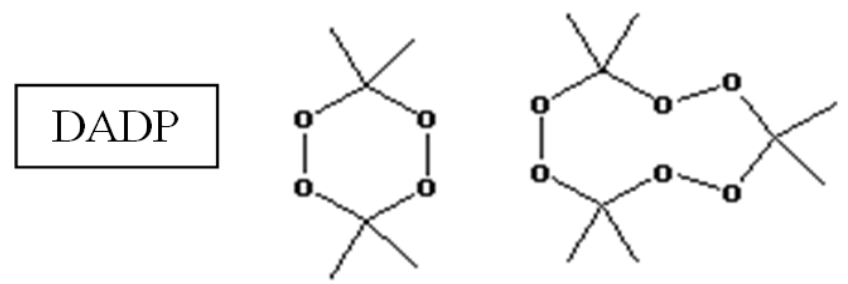

TATP

Bali et. al., have noted that research to counter the threat of organic peroxides such as triacetone triperoxide (TATP) is at times hampered by their inherent extreme sensitiveness and volatility [6].

Thermal decomposition and thermochemistry of TATP and some other organic peroxides have been investigated by various researchers [7-12]. The thermal decomposition of two peroxides, triacetone triperoxide (TATP) and hexamethylene triperoxide diamine (HMTD), was investigated as a function of pressure, temperature and atmosphere by using T-jump/FT-IR spectroscopy [7]. In an argon atmosphere at fast heating rates, the dominant decomposition product of TATP was found to be methane rather than acetone which dominates at slow heating rates. The products of TATP were different in argon and in air which implies that decomposition occurs primarily in the gas phase.

Oxley et.al., discussed methods to degrade peroxide explosives, DADP, TATP, and HMTD, chemically, at room temperature. A number of mixtures containing metals (e.g., zinc, copper) and metal salts (e.g., zinc sulfate, copper chloride) were found to be 
effective, so that some capable of destroying TATP solutions in a few hours. Strong acids proved useful against solid peroxide materials [9].

In the present computational study, interaction of TATP with some group II metals has been investigated in the realm of density functional theory (DFT).

\section{Method of Calculation}

All the structures were subjected to MM2 initial geometry optimizations leading to energy minima followed by semi-empirical PM3 self-consistent fields molecular orbital (SCF MO) method $[13,14]$ at the restricted level [15]. Then, the structure optimizations have been achieved within the framework of Hartree-Fock (HF) and finally by using density functional theory (DFT) at the level of B3LYP/6-311++G(d,p) [16,17]. The exchange term of B3LYP consists of hybrid Hartree-Fock and local spin density (LSD) exchange functions with Becke's gradient correlation to LSD exchange [18]. The correlation term of B3LYP consists of the Vosko, Wilk, Nusair (VWN3) local correlation functional [19] and Lee, Yang, Parr (LYP) correlation correction functional [20]. The normal mode analysis for each structure yielded no imaginary frequencies for the $3 \mathrm{~N}-6$ vibrational degrees of freedom, where $N$ is the number of atoms in the system which indicates that the structure of each molecule corresponds to at least a local minimum on the potential energy surface. Furthermore, all the bond lengths were thoroughly searched in order to find out whether any bond cleavage occurred or not during the geometry optimization process. All these computations were performed by using SPARTAN 06 [21].

\section{Results and Discussion}

Highly dangerous materials (explosives or toxic chemicals) sometimes need new technologies or agents in order to destroy them harmlessly. Therefore, some research continuously is devoted to that direction. Since TATP molecule possesses peroxide linkages its oxidative character is dominating so that some reducing agents are required while destroying TATP. However, the redox reaction supposed to take place has to be not so vigorous to cause any danger.

The ground state electronic configurations of the metals of present interest $\mathrm{Be}, \mathrm{Mg}$ and $\mathrm{Ca}$ are $1 \mathrm{~s}^{2} 2 \mathrm{~s}^{2},[\mathrm{Ne}] 3 \mathrm{~s}^{2}$ and $[\mathrm{Ar}] 4 \mathrm{~s}^{2}$, respectively. Their first ionization energies are 900, 736 and $590 \mathrm{~kJ} / \mathrm{mol}$., whereas the second ionization energies are 1760, 1450 and 1150 in the same sequence [22]. 
Figure 1 shows the top and side views of the structure optimized composites or their decomposed forms presently considered. As seen in the figure TATP $+\mathrm{Be}$ composite is stable and undergoes no bond cleavage during the optimization process. As seen in Figure 2, TATP $+2 \mathrm{Be}$ composite also keeps its integrity.
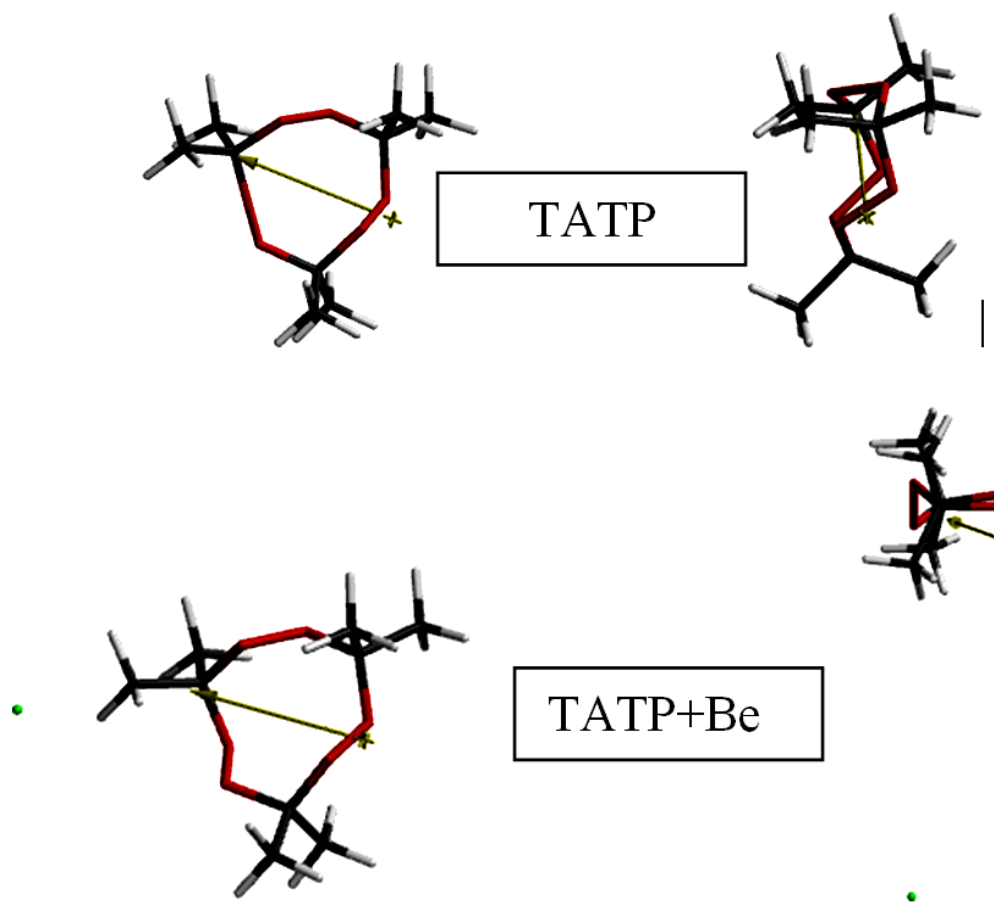

\section{$\mathrm{TATP}+\mathrm{Be}$}
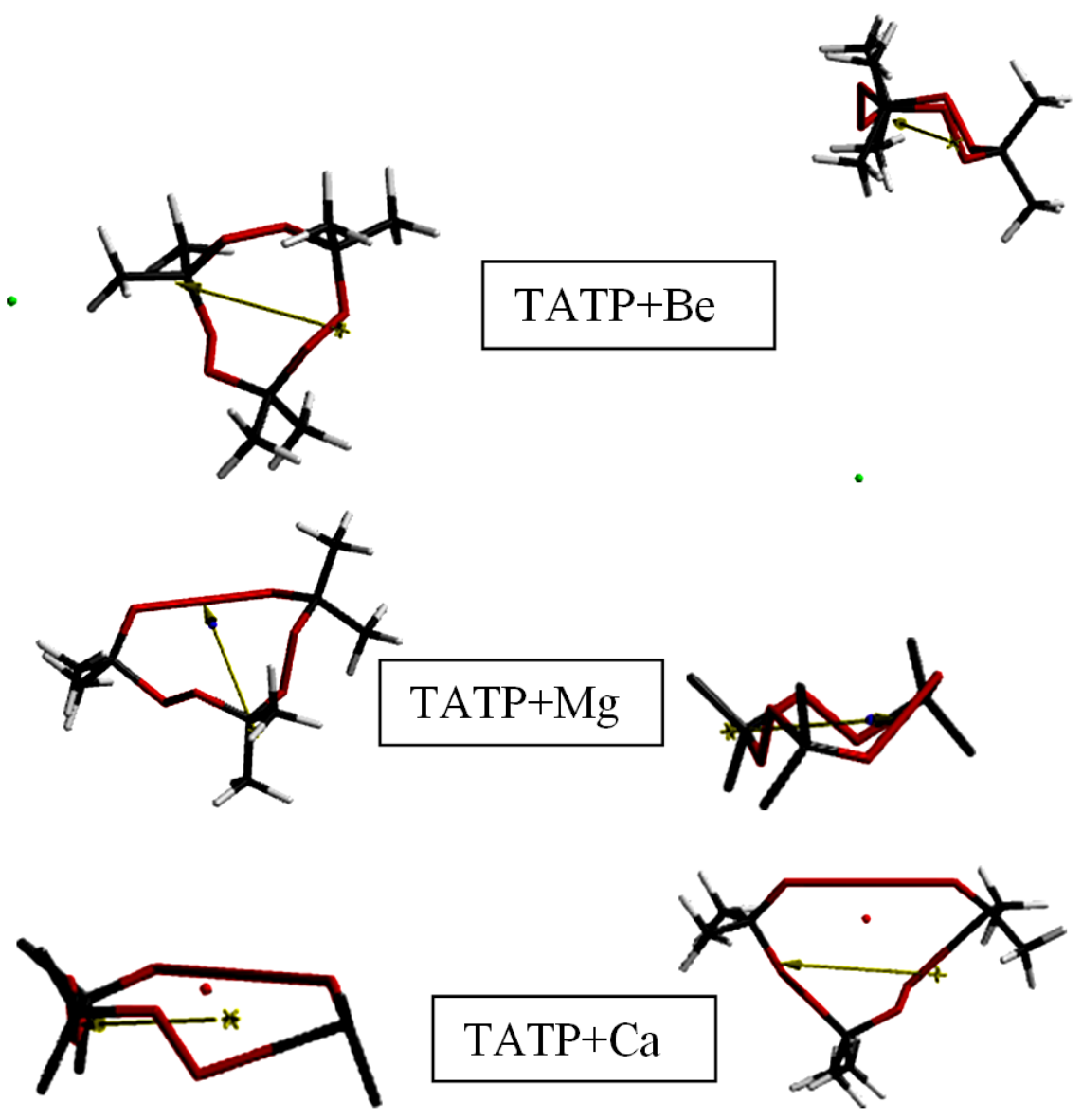

Figure 1. Optimized structures of the systems (top and side views) presently considered. 


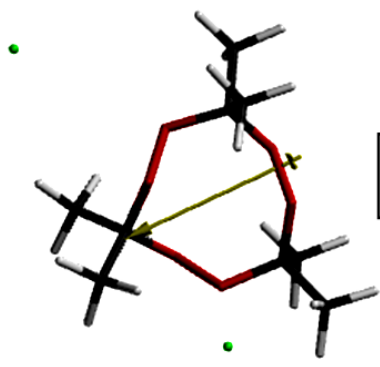

\section{$\mathrm{TATP}+2 \mathrm{Be}$}

Figure 2. Optimized structures of TATP $+2 B e$ system (top and side views).

On the other hand, TATP $+\mathrm{Mg}$ and $\mathrm{TATP}+\mathrm{Ca}$ systems undergo bond rupture(s). In the magnesium case, one of the O-O linkages is broken $(3.55 \AA)$ whereas in the calcium composite one of the peroxide bonds $(4.17 \AA$ ) as well as one of the $\mathrm{C}-\mathrm{O}$ bonds $(2.82 \AA)$ are cleaved. Figure 3 shows the bond lengths/distances in the composites considered. One of the $\mathrm{C}-\mathrm{O}$ bonds in the magnesium composite is somewhat elongated (1.63 $\AA$ ) as compared to similar types of bonds in the system.

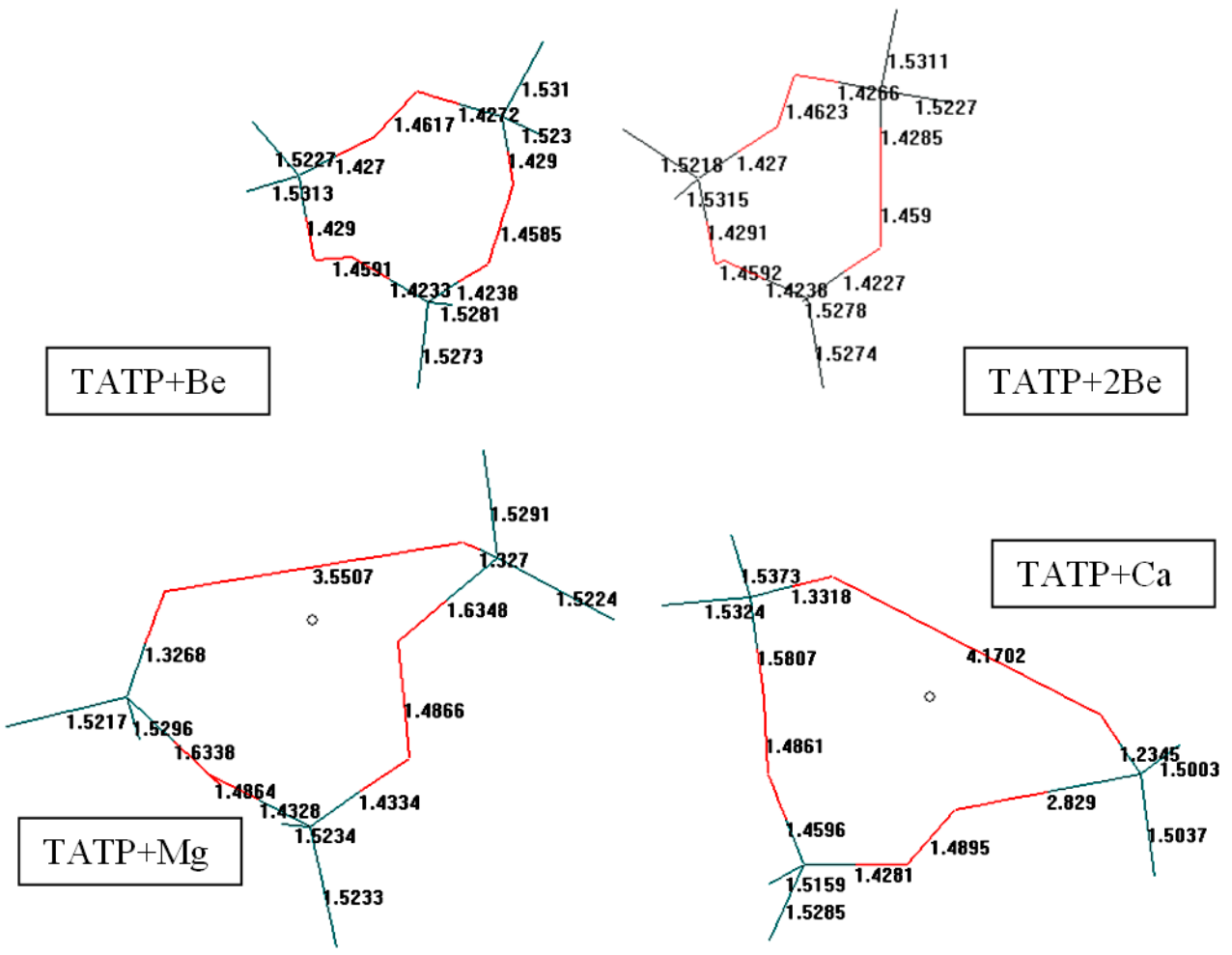

Figure 3. Bond lengths/distances ( $\AA$ ) of the systems considered (Hydrogens not shown). 
Table 1 lists some properties of the systems considered. As seen in the table, the area and volume increase in the beryllium composites as compared to TATP but the dipole moment values are the same.

Table 1. Some properties of the systems considered.

\begin{tabular}{cccccc}
\hline System & Area $\left(\AA^{\mathbf{2}}\right)$ & $\begin{array}{c}\text { Volume } \\
\left(\AA^{3}\right)\end{array}$ & $\begin{array}{c}\text { Dipole } \\
\text { moment }\end{array}$ & Ovality & Polarizability \\
\hline TATP & 250.18 & 222.42 & 0.11 & 1.41 & 57.88 \\
TATP+Be & 269.37 & 230.32 & 0.11 & 1.48 & 58.90 \\
TATP+2Be & 288.53 & 238.19 & 0.11 & 1.55 & 59.55 \\
TATP+Mg & 270.23 & 235.48 & 3.95 & 1.47 & 59.08 \\
TATP+Ca & 288.17 & 246.05 & 4.69 & 1.52 & 60.32 \\
\hline
\end{tabular}

Dipole moments in debye units.

Table 2 shows some energies of the systems presently considered.

Table 2. Some energies of the systems of consideration.

\begin{tabular}{cccc}
\hline System & $\mathbf{E}$ & $\mathbf{Z P E}$ & $\mathbf{E}_{\mathbf{C}}$ \\
\hline TATP+Be & -2152384.56 & 715.49 & -2151669.07 \\
TATP+2Be & -2190903.90 & 714.94 & -2190188.96 \\
TATP $+\mathrm{Mg}$ & -2639776.59 & 707.32 & -2639069.27 \\
TATP+Ca & -3893547.49 & 700.02 & -3892847.47 \\
\hline
\end{tabular}

Energies in $\mathrm{kJ} / \mathrm{mol}$.

Figure 4 displays the electrostatic potential charges (ESP) and electrostatic potential maps of the present concern. 

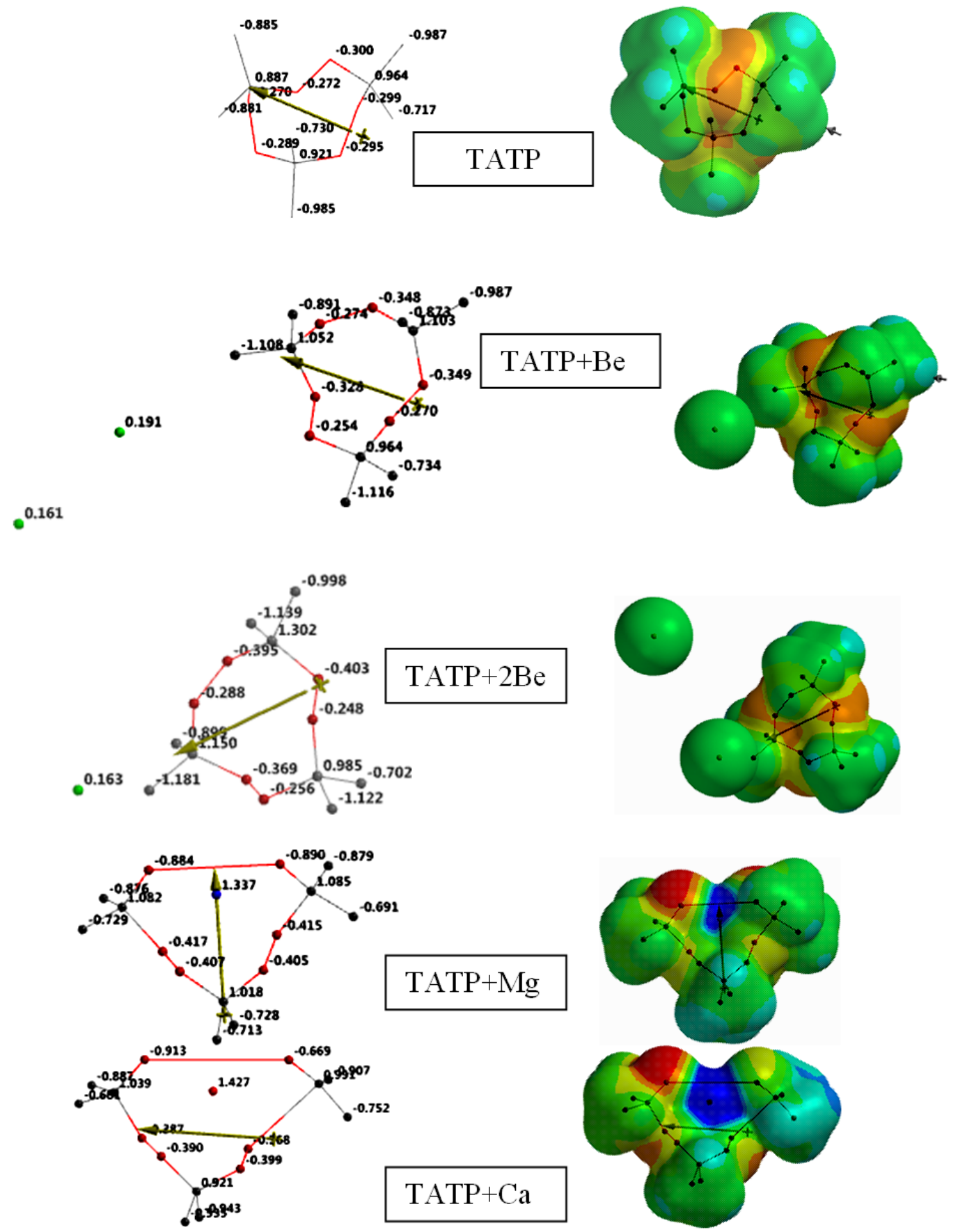

Figure 4. ESP charges and potential maps of the systems considered (Hydrogens not shown). 
Note that the ESP charges are obtained by the program based on a numerical method that generates charges that reproduce the electrostatic potential field from the entire wavefunction [21].

The charges possessed by the metallic elements considered are all positive. They are 1.91 and 1.61-1.63 for TATP $+\mathrm{Be}$ and $\mathrm{TATP}+2 \mathrm{Be}$ systems, respectively. Whereas the charges of metals in the other cases are $1.33(\mathrm{TATP}+\mathrm{Mg})$ and $1.42(\mathrm{TATP}+\mathrm{Ca})$. Note that the first ionization energies of $\mathrm{Be}, \mathrm{Mg}$ and $\mathrm{Ca}$ are 900,736 and $590 \mathrm{~kJ} / \mathrm{mol}$. [22], respectively. Namely calcium donates some electron population more favorably than magnesium and magnesium better than beryllium. Note that all the peroxide oxygens have unequal partial negative charges.

Figure 5 displays some of the molecular orbital energy levels of the systems considered. Table 5 includes the HOMO, LUMO (frontier molecular orbitals) energies and the interfrontier molecular orbital energy gap $(\Delta \varepsilon)$ values.

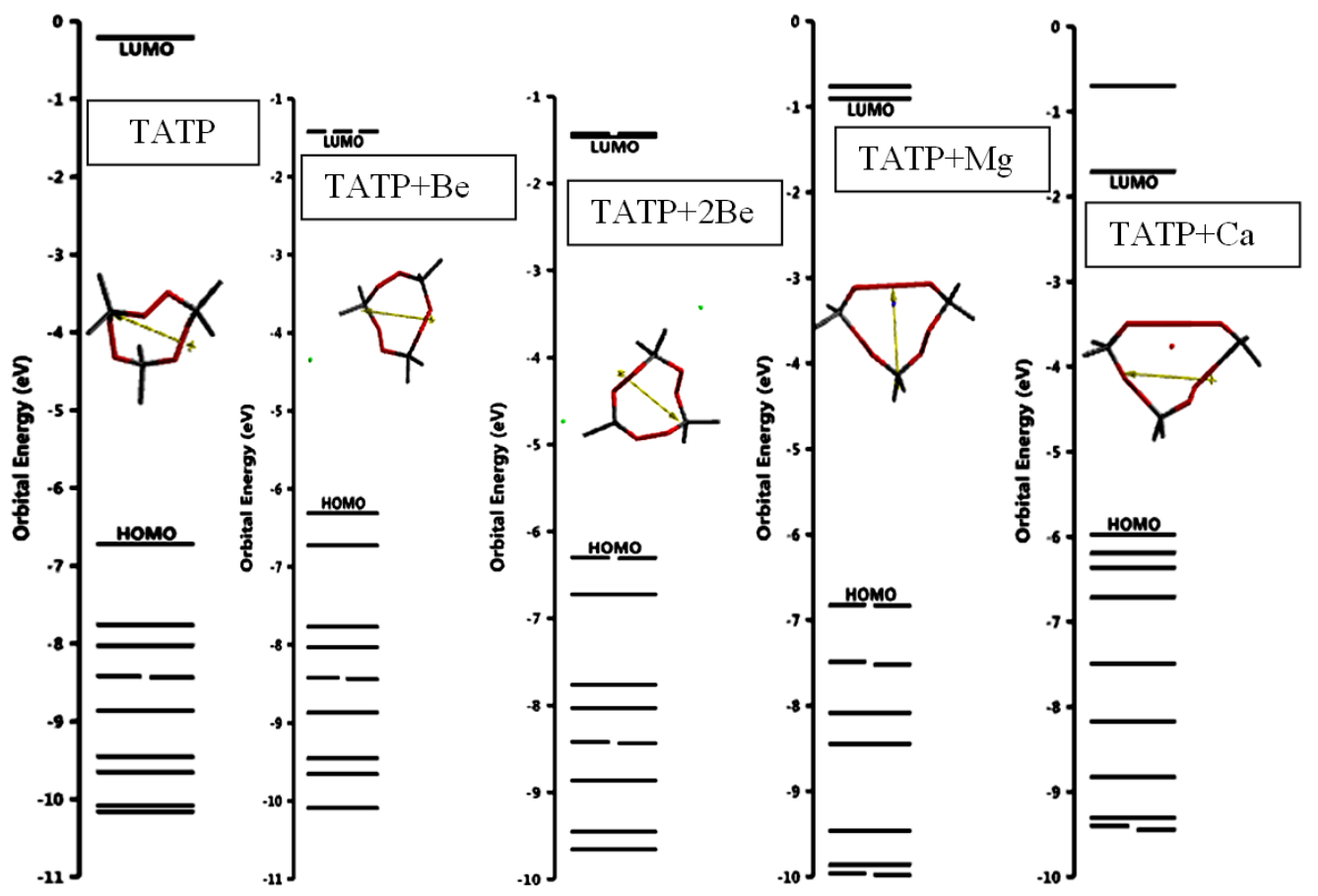

Figure 5. Some of the molecular orbital energies of the systems considered (Hydrogens not shown).

As seen in Figure 5 and Table 3, the presence of beryllium or calcium raises up whereas magnesium lowers the HOMO of the composite as compared to TATP case. 
However, note that in the $\mathrm{Mg}$ and $\mathrm{Ca}$ cases the values stand for the decomposed composites.

Table 3. The HOMO, LUMO energies and $\Delta \varepsilon$ values.

\begin{tabular}{cccc}
\hline System & HOMO & LUMO & $\boldsymbol{\Delta} \boldsymbol{\varepsilon}$ \\
\hline TATP & -648.56 & -20.88 & 627.68 \\
TATP+Be & -608.56 & -136.93 & 471.63 \\
TATP+2Be & -607.72 & -141.11 & 466.61 \\
TATP+Mg & -658.12 & -87.46 & 570.66 \\
TATP+Ca & -576.47 & -164.29 & 412.18 \\
\hline
\end{tabular}

Energies in $\mathrm{kJ} / \mathrm{mol}$.

The HOMO order is TATP $+\mathrm{Mg}<\mathrm{TATP}<\mathrm{TATP}+\mathrm{Be}<\mathrm{TATP}+2 \mathrm{Be}<\mathrm{TATP}+\mathrm{Ca}$. It seems calcium raises up the HOMO much better than magnesium and beryllium. Since the first ionization energies of $\mathrm{Be}, \mathrm{Mg}$ and $\mathrm{Ca}$ are 900,736 and $590 \mathrm{~kJ} / \mathrm{mol}$. [22], respectively. The $\mathrm{Be}$ atom cannot donate any electron population so easily. The position of Ca composite in the order seems to be odd at the first sight. However, note that in the cases of $\mathrm{Ca}$ and $\mathrm{Mg}$, the HOMO and LUMO energies are for the decomposed systems not for the composites having an intact TATP molecule. Also note that in the $\mathrm{Mg}$ case although the electrostatic charges on the oxygen atoms at the termini of ruptured O-O bond are very comparable in magnitude (suggesting a homolytic cleavage), in the case of $\mathrm{Ca}$ composite the respective charges on the broken peroxide bond is not comparable in magnitude in spite of the fact that both are negative in sign. The LUMO order within the frame of what have been said above is $\mathrm{TATP}+\mathrm{Ca}<\mathrm{TATP}+2 \mathrm{Be}<\mathrm{TATP}+\mathrm{Be}<$ $\mathrm{TATP}+\mathrm{Mg}<\mathrm{TATP}$. That means the metal partners lower the LUMO energy of TATP at different extends.

Figure 6 shows the time-dependent density functional (TDDFT) UV-VIS spectra of the systems presently considered. As expected TATP absorbs in the UV region of the spectrum because it does not have any conjugated paths to absorb at wavelengths for less energy requiring excitations $[23,24]$. So do the beryllium composites of it. However for the decomposed composites of TATP the situation is different. Although, the decomposed TATP $+\mathrm{Mg}$ absorbs in the UV region, the decomposed TATP $+\mathrm{Ca}$ spectrum 
exhibits a bathochromic effect $[24,25]$ to longer wavelengths in the UV and partly in the VIS regions. Note that in the case of TATP $+\mathrm{Ca}$ two bonds, $\mathrm{O}-\mathrm{O}$ and $\mathrm{C}-\mathrm{O}$ bonds, have been broken which may have different type interactions to shift the absorption to longer $\lambda_{\max }$ values.

Figure 7 displays the HOMO and LUMO patterns of the systems of present concern. In TATP molecule the HOMO orbital has $\pi$-type symmetry in between the oxygen atoms but they all have antibonding interaction. In the LUMO orbital the interaction between the oxygen atoms is again antibonding but this time $\sigma$-type. In the beryllium composite, the organic component does not contribute to the HOMO and LUMO. A similar situation occurs in the case of TATP $+2 \mathrm{Be}$. The contributions to the frontier molecular orbitals are only from the $\mathrm{Be}$ atom. In the decomposed magnesium composite $\mathrm{Mg}$ atom does contribute neither to the HOMO nor the LUMO. In the case of decomposed calcium composite, the metal atom has some small contribution to both the HOMO and LUMO.

Figure 8 shows the calculated IR spectra of the systems considered. The calculated IR spectrums of both beryllium composites are similar to the spectrum of TATP. The magnesium case differing from the spectra of both TATP and beryllium composites indicates that how great structural perturbations have occurred. In the series considered the extreme situation is associated with TATP $+\mathrm{Ca}$ case. The strong peak at $1693 \mathrm{~cm}^{-1}$ belongs to $\mathrm{C}-\mathrm{O}$ vibration. The corresponding bond length is $1.23 \AA$ (suggesting a $\mathrm{C}=\mathrm{O}$ bond) which is shorter than the $\mathrm{C}-\mathrm{O}$ bond at the other side of the broken $\mathrm{O}-\mathrm{O}$ bond (1.33 $\AA$ ). All the other C-O bond lengths vary in between 1.43-1.58 A. Note that cleavage of O$\mathrm{O}$ bond is accompanied by the cleavage of $\mathrm{C}-\mathrm{O}$ bond at the same carbon atom. All these suggest that a redox reaction should have occurred to liberate a formaldehyde molecule (or alike specie) by the action of $\mathrm{Ca}$ atom.

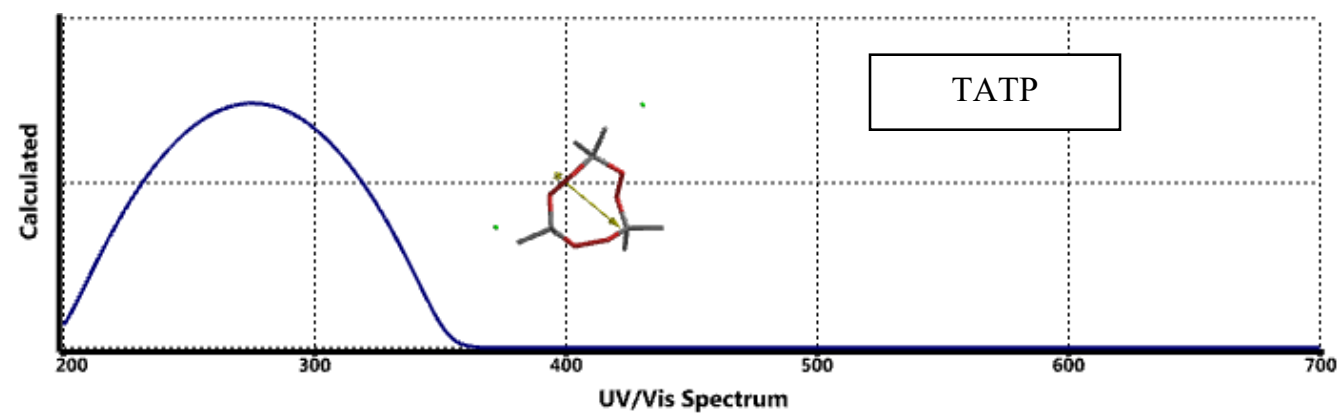



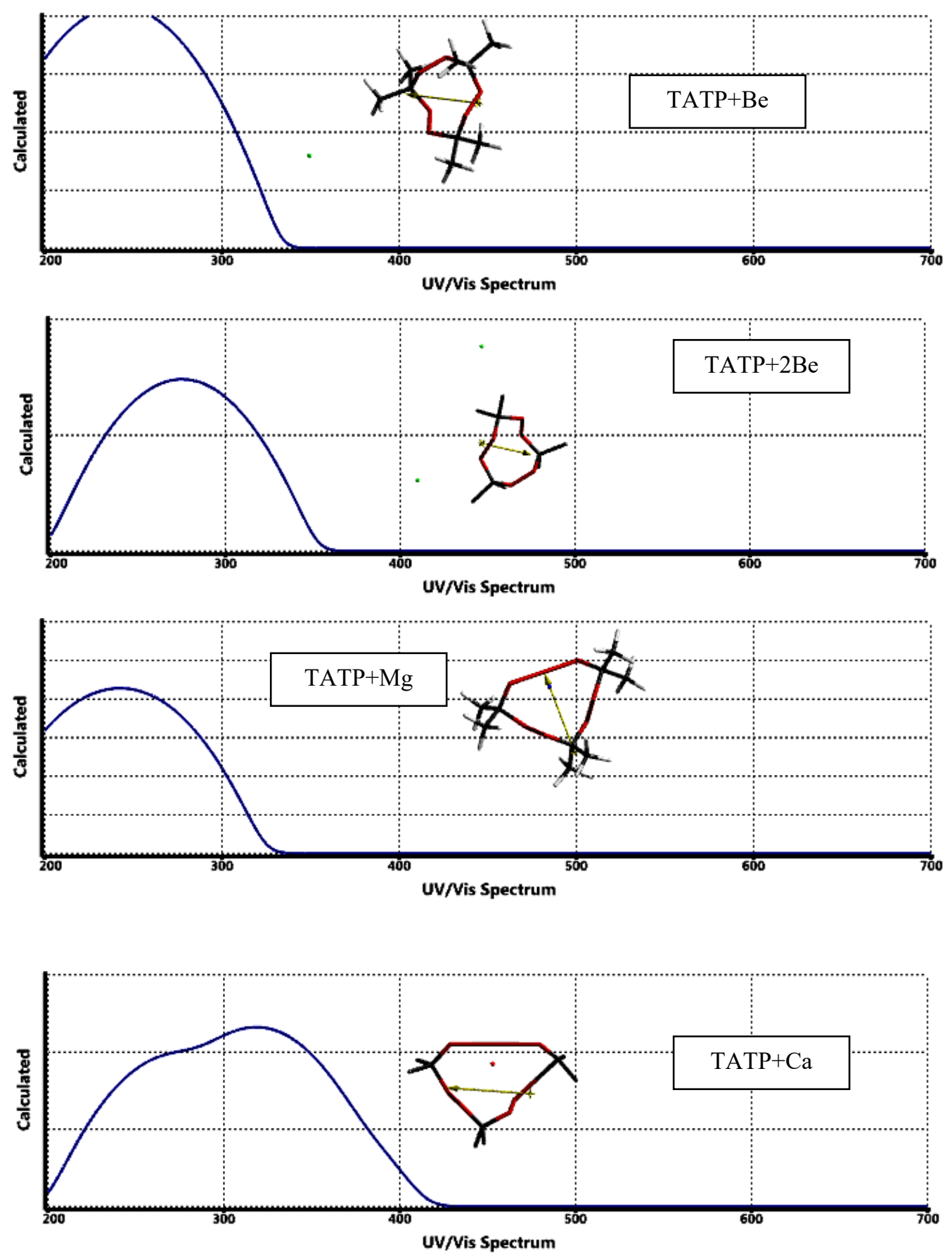

Figure 6. UV-Vis spectra of the systems considered. 

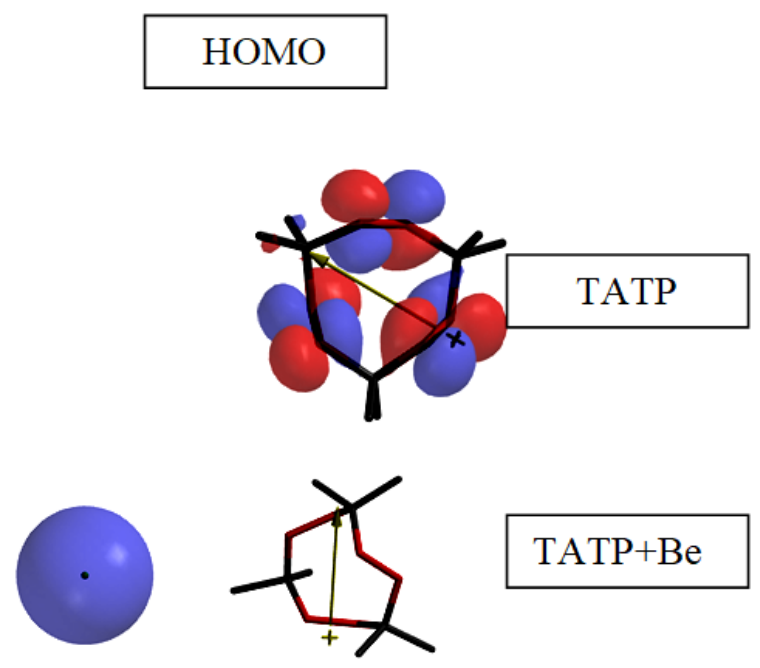

$\mathrm{TATP}+\mathrm{Be}$

$\mathrm{TATP}+2 \mathrm{Be}$

\section{LUMO}

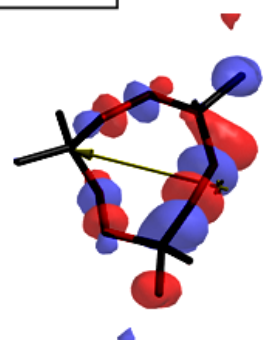

$\triangle$
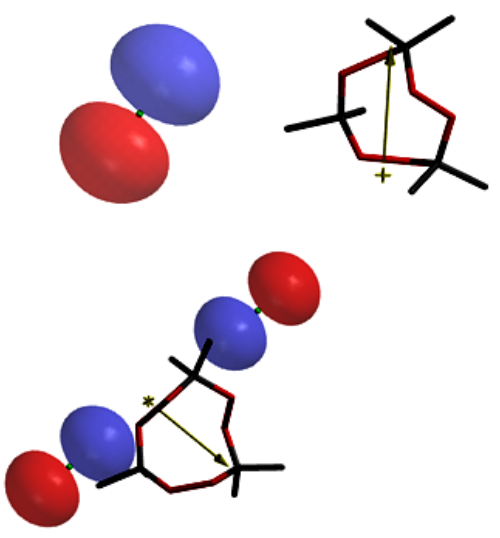

3
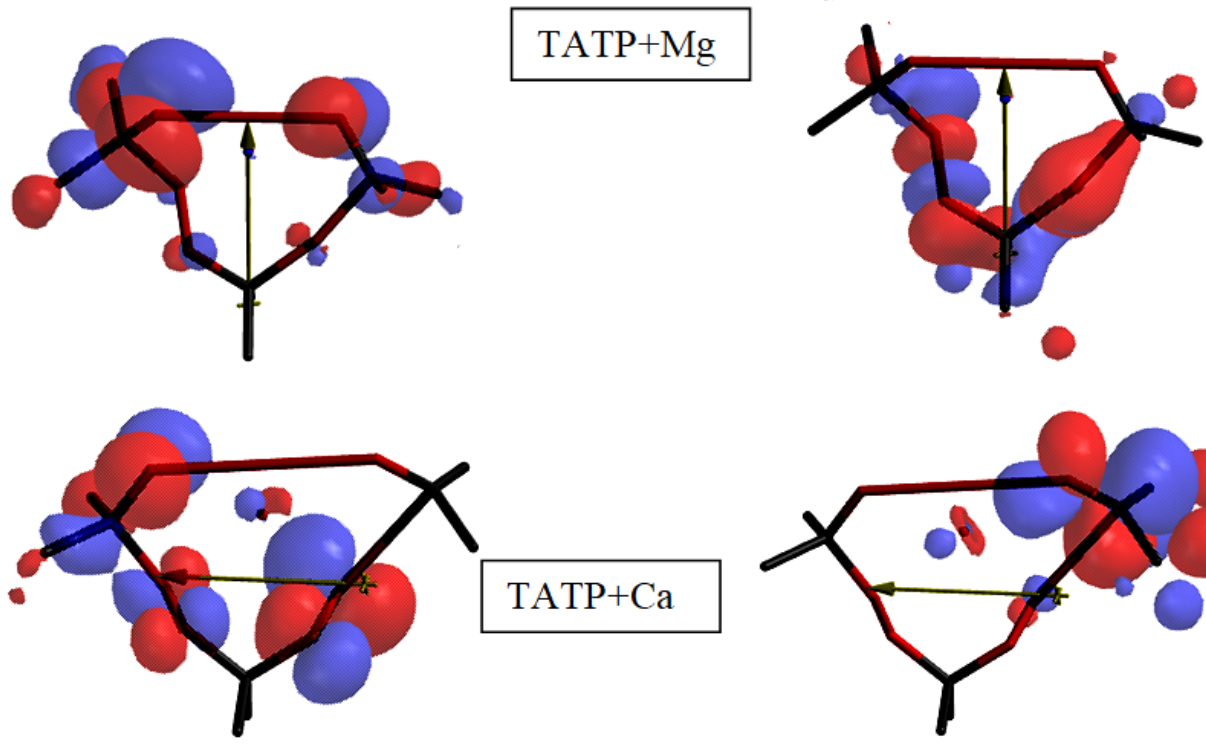

\section{$\mathrm{TATP}+\mathrm{Ca}$}

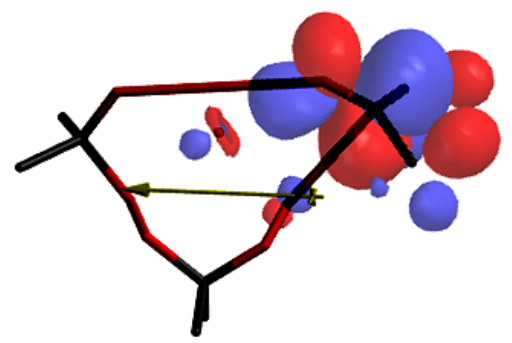

Figure 7. The HOMO and LUMO patterns of the systems of present concern. 

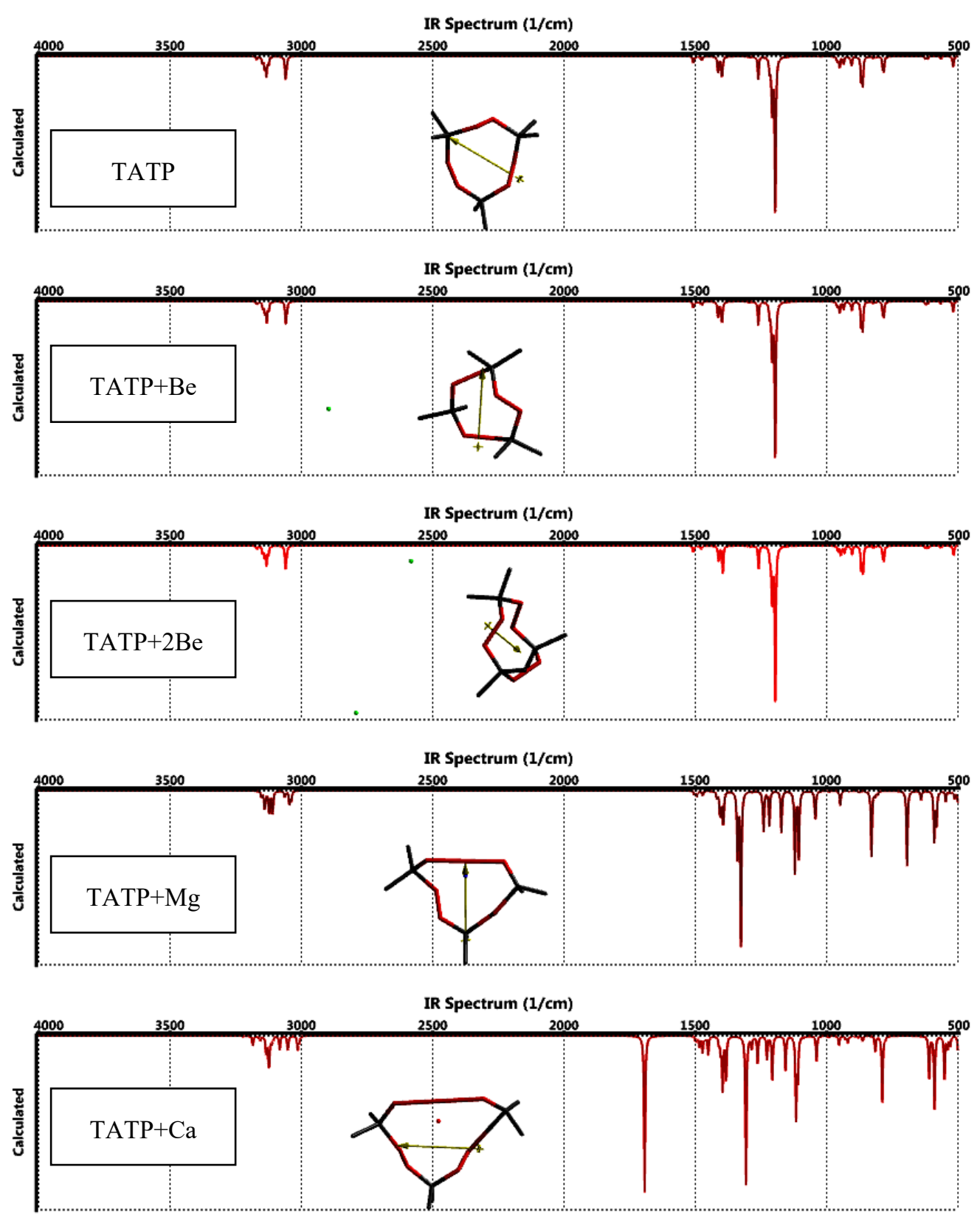

Figure 8. The calculated IR spectra of the systems considered.

Figure 9 shows the local ionization potential maps of the systems considered. In the local ionization potential map conventionally red regions on the density surface indicate areas from which electron removal is relatively easy, meaning that they are subject to electrophilic attack. On the other hand, regions having blue color represent areas where 
ionization is relatively difficult. Thus the figure indicates that $\mathrm{Be}$ atom is the relatively suitable site for electrophilic attack while itself being oxidized. Whereas, $\mathrm{Mg}$ and $\mathrm{Ca}$ atoms in the decomposed composites are potential sites for nucleophilic attack because they are already in the oxidized state.

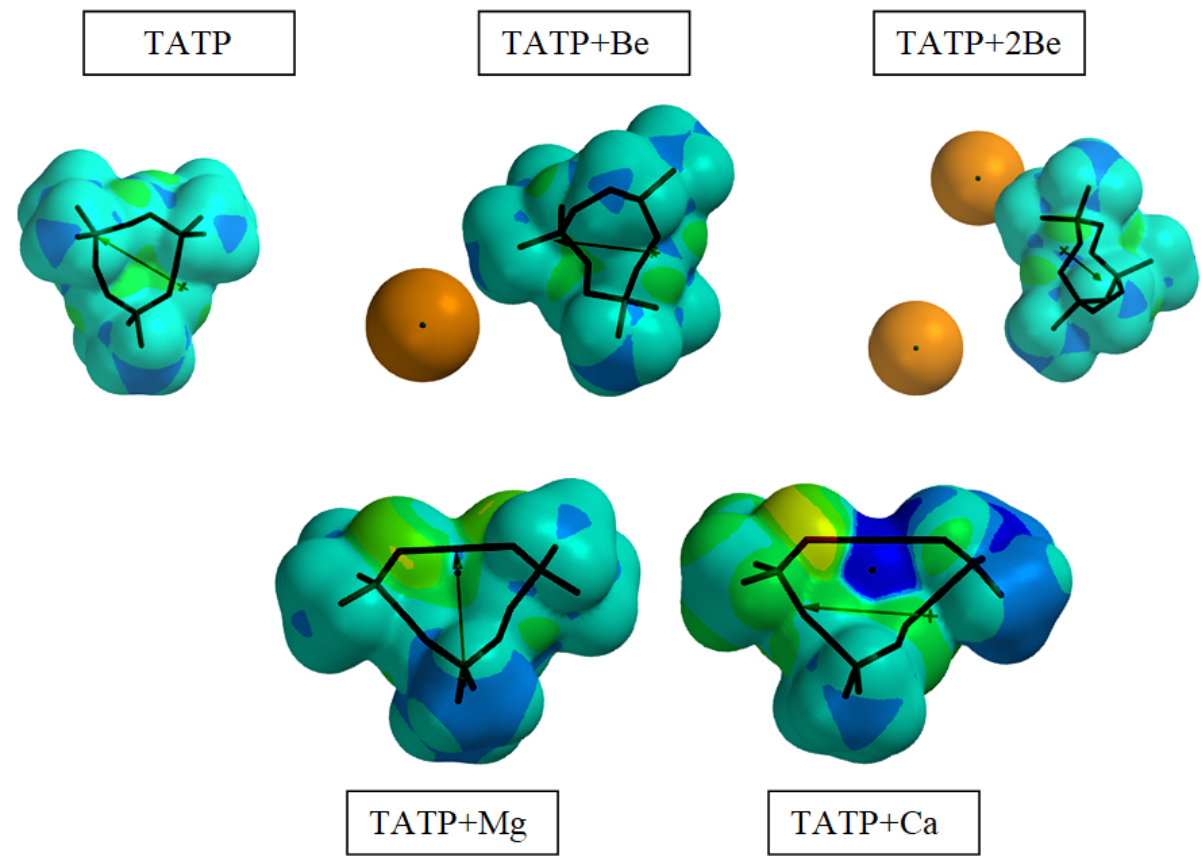

Figure 9. Local ionization potential maps of the systems considered.

\section{Conclusion}

The present density functional investigation (within the constraints of the theory and basis set applied) of TATP composites with some group II metals (Be, $\mathrm{Mg}$ and $\mathrm{Ca}$ ) has revealed that the organic component remains intact in the presence of beryllium, even the presence of two $\mathrm{Be}$ atoms, whereas in the cases of $\mathrm{Mg}$ and $\mathrm{Ca}$ composites, one of the $\mathrm{O}$ $\mathrm{O}$ bonds is ruptured. In the TATP $+\mathrm{Ca}$ composite, not only the peroxide bond but also one of the $\mathrm{C}-\mathrm{O}$ bonds incident to the same carbon atom has been broken. The spectral and bond length calculations have revealed that that particular carbon atom turns into formaldehyde (or alike specie). These decompositions involve transfer of some electron population from the metal atom to TATP molecule. In the case of calcium composite after the main redox reaction some further reaction(s) occur to produce a formaldehyde like specie to be eliminated. 


\section{References}

[1] Wolffenstein, R. (1895). Ueber die Einwirkung von Wasserstoffsuperoxyd auf Aceton und Mesityloxyd. Ber. Dtsch. Chem. Ges., 28(2), 2265-2269. https://doi.org/10.1002/cber.189502802208

[2] Matyas, R., \& Pachman, J. (2010). Study of TATP: Influence of reaction conditions on product composition. Propellants Explos. Pyrotech. 35, 31-37. https://doi.org/10.1002/prep.200800044

[3] Jiang, H., Chu, G., Gong, H., \& Qiao, Q. (1999). Tin chloride catalyzed oxidation of acetone with hydrogen peroxide to tetrameric acetone peroxide. J. Chem. Res., 28(4), 288-289. https://doi.org/10.1039/A809955C

[4] Stiasny, B.W. (2016). Investigation of organic peroxides and their properties as energetic materials. Ph.D. Dissertation. Ludwig Maximilian University of Munich. Munich, Germany.

[5] Bulatov, V., Reany, O., Grinko, R., Schechter, I., \& Keinan, E. (2013). Time-resolved, laser initiated detonation of TATP supports the previously predicted non-redox mechanism, Phys. Chem. Chem. Phys., 15, 6041-6048. https://doi.org/10.1039/c3cp44662j

[6] Bali, M.S., Wallace, L., Day, A.I., \& Armitt, D. (2014). Cyclic pentanone peroxide: Sensitiveness and suitability as a model for triacetone triperoxide. Journal of Forensic Sciences, 59, 936-942. https://doi.org/10.1111/1556-4029.12439

[7] Hiyoshi, R.I., Nakamura, J., \& Brill, T.B. (2007). Thermal decomposition of organic peroxides TATP and HMTD by T-Jump/FTIR spectroscopy. Propellants Explos. Pyrotech., 32(2), 127-134. https://doi.org/10.1002/prep.200700002

[8] Sinditskii, V.P., Kolesov, V.I., Egorshev, V.Yu. Patrikeev, D.I., \& Dorofeev, O.V. (2014). Thermochemistry of cyclic acetone peroxides. Thermochimica Acta, 585, 10-15. https://doi.org/10.1016/j.tca.2014.03.046

[9] Oxley, J., Smith, J.L., Huang, J., \& Luo, W. (2009). Destruction of peroxide explosives. Journal of Forensic Sciences, 54(5), 1029-1033.

https://doi.org/10.1111/j.1556-4029.2009.01130.x

[10] Oxley, J.C., Smith, J.L., \& Chen, H. (2002). Decomposition of a multi-peroxidic compound: triacetone triperoxide (TATP). Propellants, Explosives, Pyrotechnics, 27, 209-216.

https://doi.org/10.1002/1521-4087(200209)27:4<209::AID-PREP209>3.0.CO;2-J

[11] Dubnikova, F., Kosloff, R., Almog, J., Zeiri, Y., Boese, R., Itzhaky, H., Alt, A., \& 
Keinan, E. (2005). Decomposition of triacetone triperoxide is an entropic explosion. $J$. Am. Chem. Soc., 127(4), 1146-1159. https://doi.org/10.1021/ja0464903

[12] Tsaplev, Y.B. (2012). Decomposition of cyclic acetone peroxides in acid media. Kinet. Catal. 53, 521-524. https://doi.org/10.1134/S0023158412050163

[13] Stewart, J.J.P. (1989). Optimization of parameters for semi empirical methods I. J. Comput. Chem. 10, 209-220. https://doi.org/10.1002/jcc.540100208

[14] Stewart, J.J.P. (1989). Optimization of parameters for semi empirical methods II. J. Comput. Chem. 10, 221-264. https://doi.org/10.1002/jcc.540100209

[15] Leach, A.R. (1997). Molecular Modeling. Essex: Longman.

[16] Kohn, W., \& Sham, L.J. (1965). Self-consistent equations including exchange and correlation effects. Phys. Rev., 140, 1133-1138.

https://doi.org/10.1103/PhysRev.140.A1133

[17] Parr, R.G., \& Yang, W. (1989). Density functional theory of atoms and molecules. London: Oxford University Press.

[18] Becke, A.D. (1988). Density-functional exchange-energy approximation with correct asymptotic behavior. Phys. Rev. A, 38, 3098-3100.

https://doi.org/10.1103/PhysRevA.38.3098

[19] Vosko, S.H., Vilk, L., \& Nusair, M. (1980). Accurate spin-dependent electron liquid correlation energies for local spin density calculations: a critical analysis. Can. J. Phys., 58, 1200-1211. https://doi.org/10.1139/p80-159

[20] Lee, C., Yang, W., \& Parr, R.G. (1988). Development of the Colle-Salvetti correlationenergy formula into a functional of the electron density. Phys. Rev. B, 37, 785-789. https://doi.org/10.1103/PhysRevB.37.785

[21] SPARTAN 06 (2006). Wavefunction Inc. Irvine CA, USA.

[22] Stark, J.G., \& Wallace, H.G. (1982). Chemistry data book. London: J. Murray.

[23] Atkins, P., \& de Paula, J. (2002). Atkins' physical chemistry. Oxford: Oxford Press.

[24] Streitwieser, A. (1961). Molecular orbital theory for organic chemists. New York: Wiley.

[25] Fleming, I. (1973). Frontier orbitals and organic reactions. London: Wiley. 